TITLE:

\title{
A study of magnetic particles' behavior by magneto-Cosserat continuum theory
}

$\operatorname{AUTHOR}(S):$

Kotera, H; Kitahara, H; Shima, S

\section{CITATION:}

Kotera, $\mathrm{H}$...[et al]. A study of magnetic particles' behavior by magneto-Cosserat continuum theory. IEEE TRANSACTIONS ON MAGNETICS 2000, 36(4): 1403-1406

\section{ISSUE DATE:}

2000-07

URL:

http://hdl.handle.net/2433/39990

\section{RIGHT:}

(c)2000 IEEE. Personal use of this material is permitted. However, permission to reprint/republish this material for advertising or promotional purposes or for creating new collective works for resale or redistribution to servers or lists, or to reuse any copyrighted component of this work in other works must be obtained from the IEEE. 


\title{
A Study of Magnetic Particles' Behavior by Magneto-Cosserat Continuum Theory
}

\author{
Hidetoshi Kotera, Harunori Kitahara, and Susumu Shima
}

\begin{abstract}
By implementing the magneto-Cosserat continuum theory in FEM scheme, it is possible to simulate magnetic alignment in powders with magnetic anisotropy during compaction in an applied magnetic field. We thereby analyze the relationship between the direction of magnetic field and compaction process, such as cross compaction and parallel compaction. The rotation of the axes is thus calculated at various conditions of applied magnetic field. In our previous study, Cosserat constant $\beta$ was given arbitrarily. The method to calculate the Cosserat constant $\beta$ is proposed and its effect on the rotation of the particles' easy axes is discussed. As a demonstration of the developed method, magnetic particles' alignment during compaction in an applied magnetic field is simulated. The calculated results are in good agreement with the experimental ones.
\end{abstract}

Index Terms-Cosserat-continuum theory, magnetic particle, powder compaction.

\section{INTRODUCTION}

$\mathbf{P}$ ERMANENT magnet is a key component for magnetic devices used in many of the electric products, such as motor and actuator. In particular, $\mathrm{Nd}-\mathrm{Fe}-\mathrm{B}$ magnet is one of the most popular materials for the permanent magnet in recent years. In producing the permanent magnet, compaction is performed in an applied magnetic field to improve its magnetic characteristics. Since anisotropic magnetic particles have their easy axes of magnetization, they are rotated and transferred by both the Maxwell stress and inter-particle Coulomb force. Understanding the particles' motion during compaction in the applied magnetic field is a key issue for improving the magnetic performance of the compact. However, it is very difficult to measure experimentally each particle's motion in the compaction die. We have, therefore, proposed particle dynamics method that is capable of calculating the magnetic particle's behavior during compaction in a magnetic field[1]. We have thereby investigated the effect of compaction process and direction of applied magnetic field on its magnetic characteristics [1]-[4].

The particle dynamics simulation obviously shows that particle's rotation is influential to the characteristics of magnetic powder. This method is useful for estimating the particles' behavior, however, it takes a long CPU time to calculate the whole powder behavior. Finite element calculation is commonly used to predict density distribution or stress distribution of powder

Manuscript received October 25, 1999. This work was supported by grant-in-aid for Scientific Research (B)(no. 08455334) by the Ministry of Education, Science, Sports and Culture. This work was also supported by grant-in-aid by the Japan society of Powder and Powder Metallurgy.

The authors are with Department of Mechanical Engineering, Kyoto University, Yoshida-Honmachi, Sakyo-ku, Kyoto, 606-8501 Japan (e-mail: \{kotera harry; shima\}@mech.kyoto-u.ac.jp).

Publisher Item Identifier S 0018-9464(00)06769-8. during compaction [5]. However, the rotation of the material is not considered in these finite element calculations. We, therefore, proposed a Magneto-Cosserat continuum theory to simulate the behavior of magnetic powder during compaction in an applied magnetic field [6]. In our previous study, we assumed the powder was isotropic, although magnetic particles have their easy axes of magnetization. Since the magnetic characteristics of the compact depend on the alignment of the easy axes, we have to treat it in the calculation.

In the present study, we propose a new model that enables us to consider the particle's easy axis of magnetization in the finite element calculation based on the Cosserat continuum theory. We calculate the relationship between particles' alignment during compaction in an applied magnetic field and compacting direction. In the Cosserat continuum theory, the Cosserat constant should be clarified. Therefore, we propose a method to calculate the Cosserat constant by the particle dynamics method, and its effect on the particles' alignment is discussed.

\section{Magneto-Cosserat CONTINUUM THEORY}

Since the stress tensor in the Cosserat continuum theory is anti-symmetric, let the symmetric part of the stress tensor be $\sigma_{i j}$ and anti-symmetric part be $\tau_{i j}^{\prime}$ Considering the Maxwell stress $T_{i j}$ as a body force, the equilibrium equation for translation reads

$$
\begin{aligned}
& \partial \sigma_{i j} / \partial x_{j}+\partial \tau_{j i}^{\prime} / \partial x_{j}+\partial T_{j i} \partial x_{j} \\
& \quad=\partial \sigma_{i j} / \partial x_{j}+\partial \tau_{j i}^{\prime} / \partial x_{j} \\
& \quad+\frac{1}{2}\left(M_{j} \cdot \partial H_{j} / \partial x_{i}-H_{j} \cdot \partial M_{j} / \partial x_{i}\right)=0 .
\end{aligned}
$$

where $H_{i}$ is strength of magnetic field, $M_{i}$ is magnetic polarization. For rotational equilibrium, we have

$$
\partial m_{j i} / \partial x_{j}+\varepsilon_{i j k} \tau_{j k}^{\prime}+\varepsilon_{i j k} M_{j} H_{k}=0,
$$

where $m_{i j}$ is the couple-stress tensor, and $\varepsilon_{i j k}$ is the alternating tensor.

We adopt a yield function for the Cosserat continuum for compressible material as

$$
\begin{gathered}
F=3 \sigma_{i j}^{\prime} \sigma_{i j}^{\prime} / 2+3 \beta \tau_{i} \tau_{i}+3 m_{i j} m_{i j} / 2 l^{2}+\left(\sigma_{m} / f\right)^{2}-\left(\rho^{n} \bar{\sigma}\right)^{2} \\
f=1 / a(1-\rho)^{m}, \quad \tau_{i}=\varepsilon_{i j k} \tau_{j k}^{\prime} / 2
\end{gathered}
$$

where

$\rho \quad$ is the relative density of the powder,

$l \quad$ is representative length for the rotation,

$\beta \quad$ is the Cosserat constant, and

$\sigma_{i j}^{\prime} \quad$ is deviatoric stress of symmetric part of the stress [6], [7].

If $\beta=0$, the effect of the anti-symmetric shear stress becomes zero and the yield function is coincident with the conventional 


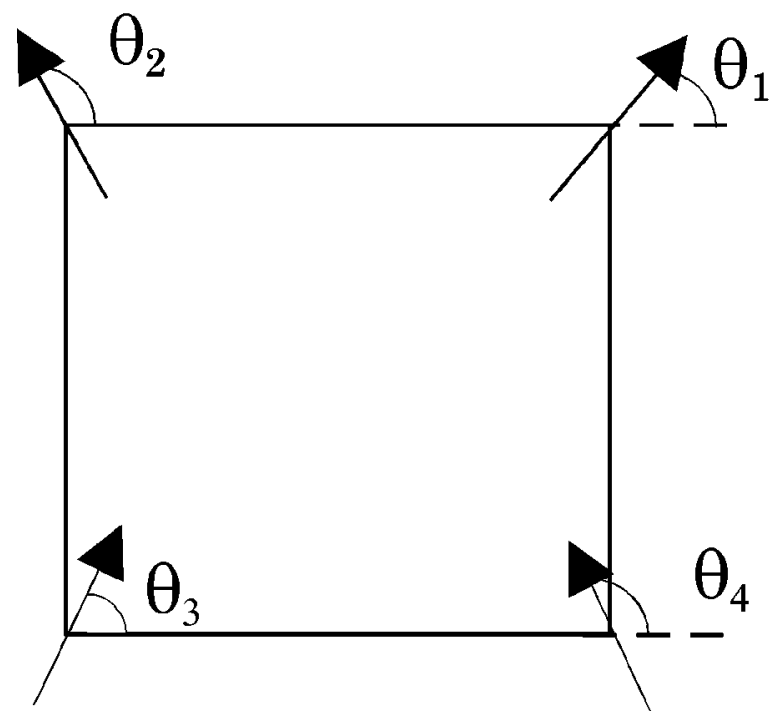

Fig. 1. Particle model at finite element node.

one [7]. $\sigma_{m}$ in Eq. (3) is the hydrostatic stress. $\bar{\sigma}$ is equivalent to the flow stress in the ordinary plasticity, but is proposed to be a constant and $n$ is a hardening parameter of compact, both of which are determined by experiment. In the present case, $l$ may refer to the particle size, because the couple stress $m_{i j}$ represents the moment which makes the magnetic particles rotate.

By the usual procedure for the finite element method, we have the variational principle from equations (1) through (3) using the Gauss divergence theorem

$$
\begin{aligned}
\Phi= & \int_{V} \dot{W} d V+\int_{S_{F}} n_{j}\left(\sigma_{j i}+\tau_{j i}^{\prime}\right) u_{i} d s+\int_{S_{M}} n_{j} m_{j i} \omega_{i} d s \\
& -\int_{V}\left(\frac{1}{2}\left(M_{k} \frac{\partial H_{k}}{\partial x_{i}}-H_{k} \frac{\partial M_{k}}{\partial x_{i}}\right) u_{i}+\varepsilon_{i j k} M_{j} H_{k} \delta \omega_{i}\right) d V .
\end{aligned}
$$

where $\dot{W}$ is strain energy rate perunit volume of a body under going plastic deformation in a magnetic field. $\dot{W}$ is defined as

$$
\dot{W}=\sigma_{j i} \dot{\varepsilon}_{i j}+2 \tau_{i}\left(\varepsilon_{i j k} \dot{r}_{j k} / 2-\omega_{i}\right)+m_{j i} \dot{\kappa}_{i j},
$$

where $\dot{\varepsilon}_{j i}$ is the strain rate, and $\dot{r}_{i j}$ rotation in the material and $k y$ is curvature rate

$$
\dot{\kappa}_{i j}=\partial \omega_{i} / \partial x_{j} .
$$

Velocity $\left(u_{i}, \omega_{i}\right)$ that minimizes $\Phi$ in Eq. (4) gives the solution.

In the finite element method, we assume that $\omega i$ is given by its nodal value, see Fig. 1. In this study, we deal with planes train case. We have

$$
\sigma=\boldsymbol{D} \varepsilon
$$

where

$$
\begin{aligned}
\sigma & =\left(\sigma_{x}, \sigma_{y}, \sigma_{x y}, \tau_{z}, m_{x z} / l, m_{y z} / l\right)^{t}, \\
\varepsilon & =\left(\dot{\varepsilon}_{x}, \dot{\varepsilon}_{y}, \dot{\varepsilon}_{x y}, \dot{\Omega}_{z}, \dot{\kappa}_{x z} l, \dot{\kappa}_{y z} l\right), \\
\dot{\Omega}_{z} & =\varepsilon_{i j k} \dot{r}_{j k} / 2-\omega_{i}
\end{aligned}
$$

$$
\begin{aligned}
\boldsymbol{D}= & \rho^{2 n-1} \overline{\bar{\sigma}} \overline{\overline{\dot{\varepsilon}}} \\
& \cdot\left[\begin{array}{cccccc}
f^{2}+4 / 9 & f^{2}-2 / 9 & 0 & 0 & 0 & 0 \\
f^{2}-2 / 9 & f^{2}+4 / 9 & 0 & 0 & 0 & 0 \\
0 & 0 & 2 / 3 & 0 & 0 & 0 \\
0 & 0 & 0 & 2 / 3 \beta & 0 & 0 \\
0 & 0 & 0 & 0 & 2 / 3 & 0 \\
0 & 0 & 0 & 0 & 0 & 2 / 3
\end{array}\right],
\end{aligned}
$$

and $\overline{\dot{\varepsilon}}$ is the equivalent strain rate.

For the case of anisotropic powder, it is necessary to introduce the easy axis in the simulation in order to estimate the magnetic characteristics of the compacts. Particle model we propose here is shown in Fig. 1. As for the ordinarily variables, angle of the easy axis for magnetization $\theta_{i}^{0}$ is given at node $i$ and $\theta_{i}^{\text {step }}$ is updated by the angular velocity as

$$
\theta_{i}^{\text {step }}=\theta_{i}^{\text {step }-1}+\omega_{i} \Delta t
$$

where step refers to the incremental step of calculation.

\section{COSSERAT CONSTANT $\beta$}

The Cosserat constant $\beta$ is the measure of the effect of the anti-symmetric stress on the yield behavior [see Eq. (3)]. Since the powder particles can be rotated more easily at a lower density than at higher, it may be assumed that the Cosserat constant is a function of the density of the powder. It is rather difficult to estimate the anti-symmetric part of the shear stress, and therefore, we calculate the relationship between the Cosserat constant and powder density with the aid of the particle dynamics method.

During compaction in the applied magnetic field, the powder is subjected to plastic deformation. The yield function $F$, defined by Eq. (3), satisfies the condition,

$$
\begin{gathered}
\frac{3}{2} \sigma_{i j}^{\prime \alpha i} \sigma_{i j}^{\prime \alpha i}+3 \beta \tau_{i}^{\alpha i} \tau_{i}^{\alpha i}+\left(3 / 2 l^{2}\right) m_{i j}^{\alpha i} m_{i j}^{\alpha i} \\
\quad+\left(\sigma_{m}^{\alpha i} / f\right)^{2}-\left(\rho^{n} \bar{\sigma}\right)^{2}=0,
\end{gathered}
$$

where $\alpha i$ refers to the direction of the applied magnetic field in the infinitesimal area.

If the density and thus the stress is constant in the infinitesimal area of the powder, the couple stress $m_{i j}$ becomes constant, thus we have

$$
\partial m_{i j} / \partial x_{j}=0
$$

According to the rotational equilibrium Eq. (2), the anti-symmetric shear stress $\tau_{i j}^{\prime}$ is written as

$$
\tau_{i j}^{\prime}=M_{j} H_{i}-M_{i} H_{j} / 2 .
$$

Since the representative length $l$ is a diameter of the powder particle, the coupe stress can be calculated as,

$$
m_{i j} m_{i j}=l^{2}|\boldsymbol{M} \times \boldsymbol{H}| \text {. }
$$

If we derive the deviatoric stress $\sigma_{i j}^{\prime}$ and the hydrostatic stress $\sigma_{m}$ for three directions of the applied magnetic field, $(\alpha 1, \alpha 2$, $\alpha 3)$, for the prescribed powder density $\rho$ by the particle dynamics method [1], the Cosserat constant $\beta$ can be calculated by solving the following equation.

$$
\begin{aligned}
& \beta=\left\{\frac{3}{2}\left({\sigma^{\prime}}_{i j}^{\alpha 3} \sigma_{i j}^{\alpha 3}-\sigma_{i j}^{\prime \alpha 2}{\sigma^{\prime}}_{i j}^{\alpha 2}\right)\right. \\
& +3\left(m_{i j}^{\alpha 3} m_{i j}^{\alpha 3}-m_{i j}^{\alpha 2} m_{i j}^{\alpha 2}\right) / 2 \ell^{2}
\end{aligned}
$$




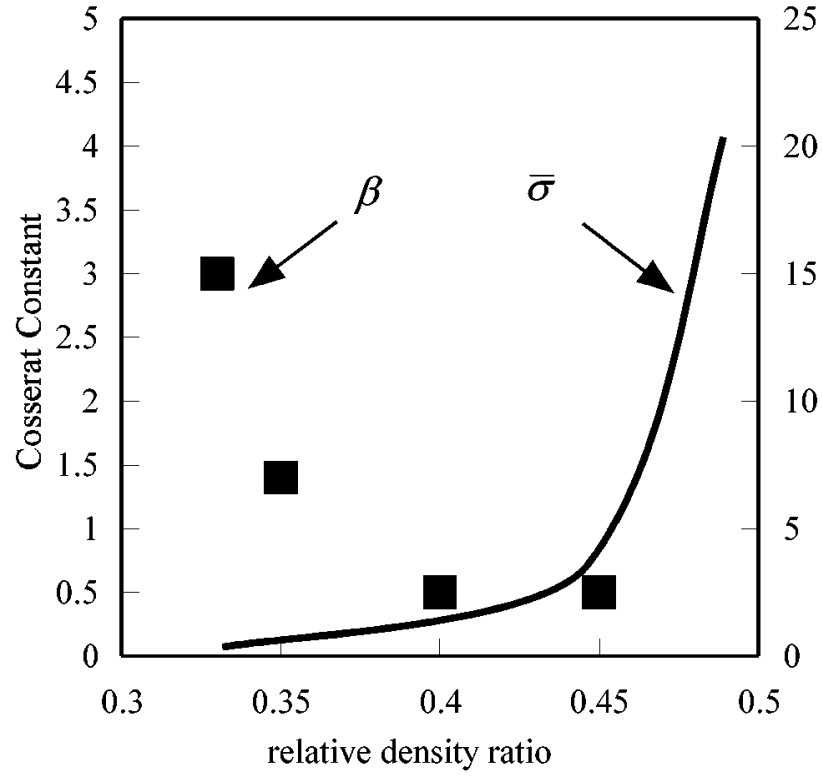

Fig. 2. Dependency of Cosserat constant $\beta$ and flow stress of $\mathrm{Ne}-\mathrm{Fe}-\mathrm{B}$ powder on relative density.

$$
\begin{aligned}
&+ {\left[3\left({\sigma^{\prime}}_{i j}^{\alpha 1}{\sigma^{\prime}}_{i j}^{\alpha 1}-{\sigma^{\prime}}_{i j}^{\alpha 2}{\sigma^{\prime}}_{i j}^{\alpha 2}\right) / 2\right.} \\
&\left.+3\left(m_{i j}^{\alpha 1} m_{i j}^{\alpha 1}-m_{i j}^{\alpha 2} m_{i j}^{\alpha 2}\right) / 2 \ell^{2}\right] \\
&\left.\left(\sigma_{m}^{\alpha 3}\right)^{2}-\left(\sigma_{m}^{\alpha 2}\right)^{2} /\left(\sigma_{m}^{\alpha 2}\right)^{2}-\left(\sigma_{m}^{\alpha 1}\right)^{2}\right\} / \\
&\left\{3\left(\tau_{i}^{\alpha 2} \tau_{i}^{\alpha 2}-\tau_{i}^{\alpha 3} \tau_{i}^{\alpha 3}\right)+3\left(\tau_{i}^{\alpha 2} \tau_{i}^{\alpha 2}-\tau_{i}^{\alpha 1} \tau_{i}^{\alpha 1}\right)\left(\sigma_{m}^{\alpha 3}\right)^{2}\right. \\
&\left.\quad-\left(\sigma_{m}^{\alpha 2}\right)^{2} /\left(\sigma_{m}^{\alpha 2}\right)^{2}-\left(\sigma_{m}^{\alpha 1}\right)^{2}\right\} .
\end{aligned}
$$

To calculate the Cosserat constant $\beta$, the particle dynamics simulation was carried out using representative 3D particle which is $0.1 \mathrm{~mm}$ long, $0.05 \mathrm{~mm}$ thick and $0.1 \mathrm{~mm}$ dia. The obtained relationship between the Cosserat constant $\beta$ and the powder density is shown in Fig. 2. Since the particles can be rotated more easily at a lower density than at higher, the Cosserat constant $\beta$ decreases with increasing density ratio. Above a density ratio of powder 0.4 , the Cosserat constant seems to be saturated to 0.4 .

In this study, the material parameters, $\bar{\sigma}, n, m$ and $a$, were measured by the simple compression test of $\mathrm{Nd}-\mathrm{Fe}-\mathrm{B}$ powder. The flow stress $\bar{\sigma}$ of magnetic powder increases with increasing the density ratio as shown in Fig. 2.

\section{RESUlTS AND DisCUSSION}

To make a permanent magnet, $\mathrm{Nd}-\mathrm{Fe}-\mathrm{B}$ or Ferrite powders are commonly used. These powders are compressed in a magnetic field in the order of $1.06 \times 10^{6} \mathrm{~A} / \mathrm{m}$. In magnet industries, there are two types of compaction process employed; parallel compaction and cross compaction. In cross compaction, the magnetic field is perpendicular to the punch stroke, while in parallel compaction, they are parallel to each other. It is commonly considered that the particles' easy axes for magnetization are aligned at a lower relative density during compaction. The mean angle of particles' easy axes in the compact of $\mathrm{Nd}-\mathrm{Fe}-\mathrm{B}$ powder measured from the direction of applied magnetic field is smaller in the cross compaction than in parallel [9].
TABLE I

Fig. 3. Magnetic axes plot before compaction (arrows refer to initial direction of easy axis at nodes).

\begin{tabular}{cl}
\multicolumn{2}{c}{ TABLE I } \\
MATERIAL PROPERTIES FOR ANALYSIS \\
material parameter & value \\
\hline initial density ratio $\rho_{0}$ & 0.35 \\
$\beta$ & 0.2 or function of density \\
representative length $\ell$ & $10 \quad$ micrometer \\
friction coefficient & 0.0 \\
$a$ & 2.5 \\
$m$ & 0.51 \\
$n$ & 2.5 \\
$M_{0}$ & $0.7 \quad \mathrm{~Wb} / \mathrm{m}^{3}$ \\
initial cell size for analysis & W1 $15 \mathrm{~mm}, \mathrm{H} 30 \mathrm{~mm}$ \\
\hline
\end{tabular}

In the present simulation, we calculated the particles' alignment during the two types of compaction by the Magneto-Cosserat continuum theory. The initial directions of the easy axes are set random as shown in Fig. 3. The frictional coefficient between the powder and the die wall was assumed to be 0.0 .

To simulate the dependency of the mean angle of the easy axis inside the compact on the process, the magnetic field is applied during compaction from powder's relative density of 0.35 up to 0.5 . Since the calculated result depends on the initial direction of particles' alignment, the calculation is carried out for eight initial conditions. The material parameters for analysis are listed in Table I.

The mean angles during compaction in two compaction processes are plotted in Fig. 4. The alignment of the easy axis is the smaller in cross compaction than in parallel. If we set the Cosserat constant $\beta=0.2$, the mean angles of particles' easy axes are larger than the results with $\beta$ as a function of density ratio. Since the Cosserat constant is a measure of the effect of anti-symmetric shear stress on the yield behavior, the particles' rotation became so small with constant $\beta$.

Experimentally, the magnetic powder, $\mathrm{Nd}-\mathrm{Fe}-\mathrm{B}$, was compressed in the applied magnetic field both by cross compaction and by parallel compaction. The average particles' alignment 


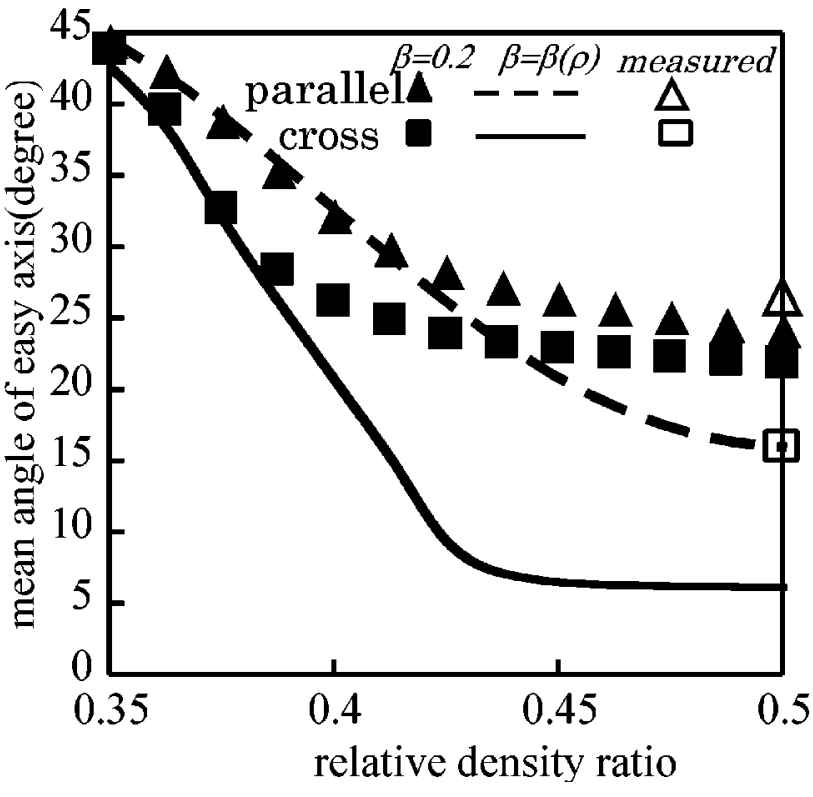

Fig. 4. Rotation of easy axis of magnetization against relative density (Magnetic field, $H=1.06 \times 10^{6} \mathrm{~A} / \mathrm{m}$ is applied from 0.35 up to 0.5 ).

$\theta_{m}$ in the compact is exerted from the measured residual flux density $B_{r}$ of the compact by $\mathrm{B}-\mathrm{H}$ tracer as,

$$
\theta_{m}=\cos ^{-1}\left(B_{r} / B_{o}\right)
$$

where $B_{0}$ is the residual flux density of $\mathrm{Nd}-\mathrm{Fe}-\mathrm{B}$ powder. The experimental results are also plotted in Fig. 4. The calculated results by the Magneto-Cosserat continuum theory agree very well with the experimental ones. Thus, the present method will be a useful tool for simulating the magnetic powder behavior, rotation of easy axes of magnetization during compaction in the applied magnetic field and also the characteristics of the compact.

By SGI origin200, it took 20 minutes to simulate the particle's alignment from powder's initial density of 0.35 up to 0.5 .

\section{CONCLUDING REMARKS}

We have developed a Magneto-Cosserat continuum theory to calculate the particle's alignment of magnetic powder during compaction in an applied magnetic field. The Cosserat constant was calculated by the particle's dynamics method. The calculated Cosserat constant was a function of the powder density. The particles' alignments during three compaction conditions in an applied magnetic field were simulated. The calculated results were in good agreement with those by particle dynamics simulation and experimental ones. We can conclude that the developed theory and method would be a useful tool for calculating the magnetic powder behavior and characteristics of the powder.

\section{ACKNOWLEDGMENT}

The authors wish to express their gratitude to Dr. Kaneko of Sumitomo Special Metal Co., Ltd. for supporting the experimentation.

\section{REFERENCES}

[1] H. Kotera, H. Kitahara, A. Onoyama, and S. Shima, "Behavior of magnetic particles in compaction," IEEE Transaction on Magnetics, vol. 32, no. 2, pp. 1616-1619, 1997.

[2] H. Kotera, H. Kitahara, and S. Shima, "Particle dynamics simulation for behavior of elliptical," Advances in Powder Metallurgy \& Particulate Materials, vol. 7, pp. 101-115, 1996.

[3] H. Kotera, A. Onoyama, and S. Shima, "Behavior of ferromagnetic granular in magnetic field and magnetic characteristics of compact by particle model," Journal of the Japan society of Powder \& Powder Metallurgy, vol. 42, pp. 645-650, 1995.

[4] H. Kotera and S. Shima, "A study of relationship between density distribution and magnetic flux in magnetic field compaction," Advances in Powder Metallurgy \& Particulate Materials, vol. 6, pp. 39-49, 1994.

[5] S. Shima, "Numerical simulation of powder behavior in compaction based on continuum and discrete models," in Proc. 1993 Powder Metallurgy World Congress, vol. 1, 1993, pp. 300-305.

[6] H. Kotera, M. Sawada, and S. Shima, "Magnetic cosserat continuum theory to simulate behavior of magnetic powder during compaction in applied magnetic field," Advances in Powder Metallurgy \& Particulate Materials, pp. 538-543, 1998.

[7] K. Mori, M. Shiomi, and K. Osakada, "Inclusion of microscopic rotation in rigid-plastic finite element analysis method using Cosserat continuum theory," International J. for Numerical Methods in Engineering, vol. 42, pp. 847-856, 1998.

[8] S. Shima, T. Inoue, M. Oyane, and K. Okimoto, "Study on compacting of metal powders (2nd Report) (Investigation of compaction criterion by three dimensional compaction)" (in Japanese), Journal of the Japan society of Powder \& Powder Metallurgy, vol. 22, pp. 257-263, 1976.

[9] Y. Kaneko and N. Ishigaki, "Recent development of high-performance NEOMAX magnets," J. Materials Engineering and Performance, no. 3 , pp. 228-223, 1994 